\title{
Fetal Anomaly Detection in Ultrasound Image
}

\author{
Athira P.K. \\ PG Student, Department of Computer Science and \\ Engineering \\ Mar Athanasius College of Engineering, \\ Kothamangalam \\ Mahathma Gandhi University-Kottayam
}

\author{
Linda Sara Mathew \\ Asst. professor Department of Computer Science \\ and Engineering \\ Mar Athanasius College of Engineering, \\ Kothamangalam \\ Mahathma Gandhi University-Kottayam
}

\begin{abstract}
Ultrasound is one of the most popular medical imaging technologies that can help a physician evaluate, diagnose and treat medical conditions. Although ultrasound imaging is generally considered good medical tool but the overall detection rate of Congenital Heart Defects (CHD) using ultrasound image remain anomic. Congenital Heart Defects are the heart problem that occurs before birth. Recognizing Congenital Heart Defects at right time is a difficult task for Physicians due to lack of subject specialists or inexperience with the previous cases or even as the children they can't express their problem in a proper way. In order to improve the diagnosis accuracy and to reduce the diagnosis time, it has become a demanding issue to develop an efficient and reliable medical Decision Support System. Hence machine learning approaches such as neural networks have shown great potential to be applied in the development of medical Decision Support System for Heart Disease. Fetal anomaly detection mainly carried out in four steps. Noise removal, segmentation, feature extraction and classification.
\end{abstract}

\section{General Terms}

Image processing

\section{Keywords}

Congenital Heart Defects, Morphological operations, Speckle noise, Ultrasound image, neural network.

\section{INTRODUCTION}

Ultrasonography is an ultrasound-based diagnostic medical imaging technique used to visualize muscles, tendons, and many internal organs, to capture their size, structure and any pathological lesions with real time tomographic images.
Ultrasound is also used to visualize fetuses during the pregnancy period and if there is a defect then doctors and parents can do emergency prenatal care. The popularity of ultrasound image is mainly due to its low cost and safety in imaging fetuses. A congenital heart defect is a disease, present at birth or occur before birth, that affecting the structure and function of the heart and major vessels. Fetal Heart Congenital Heart Defects are classified into two main categories. Holes in the heart and heart valve abnormalities. Holes in the heart can be seen between heart chambers or between major blood vessels in the heart. These holes allow oxygen-rich and oxygen-poor blood to mix. Heart valve abnormalities- If the heart valves can't open and close correctly, blood can't flow smoothly. Also valve should have correct thickness.

If these defects are identify at early stage, then proper treatment and care can be given. If it cannot be identify or treated then children have to face many problem in their future and may be lead to death. The Better Early Diagnosis for these defects is so important for early treatment or intervention. But identifying these types of Congenital Heart Defects at right time is a difficult task for Physicians due to lack of subject specialists or inexperience or the children can't express their problem in a proper way.

A very important tool for better early detection and diagnosis of these defects is ultrasound fetal echocardiography. Visual quality of the image produced from Ultrasound Fetal Echocardiography play an important role in congenital anomalies detection and diagnosis by physicians Below is a list of different types of congenital abnormality, and how likely ultrasound scanning is to identify each problem.

Table 1. Problems and chance of being seen using ultrasound image

\begin{tabular}{|c|c|c|}
\hline Problem & What the problem is & $\begin{array}{l}\text { Chance of being } \\
\text { seen }\end{array}$ \\
\hline Spina bifida & Open spinal cord & $>90 \%$ \\
\hline Anencephaly & Absence of the top of the head & $99 \%$ \\
\hline Hydrocephalus & Excess fluid within the brain & $60 \%$ \\
\hline $\begin{array}{l}\text { Major congenital heart } \\
\text { problems }\end{array}$ & & $25 \%$ \\
\hline
\end{tabular}




\begin{tabular}{|l|l|l|}
\hline Diaphragmatic hernia & $\begin{array}{l}\text { A defect in the muscle which separates the chest and } \\
\text { abdomen }\end{array}$ & $60 \%$ \\
\hline Exomphalos/gastroschisis & Defects of the abdominal wall & $90 \%$ \\
\hline Major kidney problems & Missing or abnormal kidneys & $85 \%$ \\
\hline Major limb abnormalities & Missing or abnormal kidneys & $90 \%$ \\
\hline Cerebral palsy & Spasticity & Never seen \\
\hline
\end{tabular}

But the overall detection rate of CHD remain anomic; the chance of being seen of CHD is nearly $25 \%$. . In order to improve the diagnosis accuracy and to reduce the diagnosis time, it has become a demanding issue to develop an efficient and reliable medical Decision Support System to support yet and still increasingly complicated diagnosis decision process. Proposed methods introduce a system to identify congenital heart defects to improve pregnancy outcomes and increase their detection.

\section{RESEARCH ELABORATION}

Congenital Heart Disease (CHD) is one of the major causes of deaths in children. A proper diagnosis can avoid such problem. Unfortunately, it cannot be recognize at early stage or from ultrasound image. A system for automated medical diagnosis from ultrasound image would enhance the accuracy of the diagnosis and reduce the cost effects. The proposed system is designed and developed by using MATLAB's GUI.

In diagnosis of diseases Ultrasonic devices are frequently used by healthcare professionals. Visual quality of ultrasound image had significant effect of identifying defect. The main problem during diagnosis is the distortion of visual signals obtained which is due to the consequence of the coherent of nature of the wave transmitted. These distortions are termed as 'Speckle Noise'. Speckle, in both cases, is primarily due to the interference of the returning wave at the transducer aperture. Because of the finite resolution, at any time receiving from a distribution of scatterers within the resolution cell. These scattered signals add coherently; that is, they add constructively and destructively depending on the relative phases of each scattered waveform. Speckle noise results from these patterns of constructive and destructive interference shown as bright and dark dots in the image. it reduces image contrast and degrades boundaries of structures. As a first step of identifying CHD is removal of speckle noise from ultrasound image. This is done based on patch-based Wiener filter.

After removing the speckle noise segmentation is done. Segmentation can be done using morphological operation. Segmentation is one of the most critical aspects of digital image processing. Morphology is a technique of image processing based on shapes. It involves partitioning or grouping objects in an image either for identification or analysis. There are many methods for achieving image segmentation, many of which are application-specific. A structuring element is a shape mask used in the basic morphological operation. The structuring element is a small binary image, i.e. a small matrix of pixels, each with a value of zero or one. The matrix dimensions specify the size of the structuring element. The pattern of ones and zeros specifies the shape of the structuring element. An origin of the structuring element is usually one of its pixels, although generally the origin can be outside the structuring element. There are many kinds of structuring element: Disk-shaped, Diamond-shaped, Ball-shaped, Square-shaped, Flat linear with length LEN, arbitrary with the specified neighborhood. Applying which structuring element is depending upon the image and requirements.

IM2 = imopen(IM,SE) performs morphological opening on the grayscale or binary image IM with the structuring element SE. The argument SE must be a single structuring element object, as opposed to an array of objects. Then this segmented fetal heart is used for feature extraction. A feature is a significant piece of information extracted from an image which provides more detailed understanding of the image. The gray level co-occurrence matrix (GLCM) are used for features extraction from the segmented image. After creating the GLCMs, several statistics are derived. These statistics provide information about the texture of an image. Normalization, energy, correlation, sum of variances, inverse difference moment, entropy, correlation $1 \& 2$, sum average, sum entropy, sum variance, difference variance, contrast, difference entropy are the statistics used. These features/statistics act as the basis for classification process and this classification is done with the help of Artificial neural network(ANN).

The input data for the network is obtained from the feature extracted from the images, while the output for the network will be the classification of the image. There are 13 input neurons and 3 output neurons one is for normal category other two is for two abnormal category.

\section{SYSTEM ARCHITECTURE}

The system presents four basic steps. The first step is collecting ultrasound images and denoising. The second step is image segmentation and the third step contains extraction of featur .The final step is the classification of disease with the help of neural network. Figure 1 illustrates a block diagram. The images are collected from fetal ultrasound image gallery. The original image format is jpeg and varies in size and contrast. So pre-processing is done before noise removal. Cropping and resizing are main pro-processing involved. Now denoising is done to remove noise from image. Removal of speckle noise is done based on patch-based Wiener filter. 
Segmentation by morphological operation is done to the denoised image. The morphological opening operation is performed with a structuring element. Here use periodicline structuring element. $\mathrm{SE}=\mathrm{SE}=\operatorname{strel}($ 'periodicline', $\mathrm{P}, \mathrm{V})$. Main advantage of morphological operation is their speed and simplicity. Next step is the feature extraction and classification. After the morphological operation set of features is extracted from the image withn the help of gray level cooccurrence matrix is used. Normalization, energy, correlation, sum of variances, inverse difference moment, entropy, correlation $1 \& 2$, sum average, sum entropy, sum variance, difference variance, contrast, difference entropy, are the features used. Based on this values image is classified. For this classification feedforward with back propagation neural network is used. There are 13 neurons in the input layer corresponding to 13 features and 3 neurons in the output layer corresponding to normal category and two abnormal category(hole in heart and defect in valve). Hidden layer is set to 20. After the training of the neural network real word example is gave. Finally the performance is calculated.

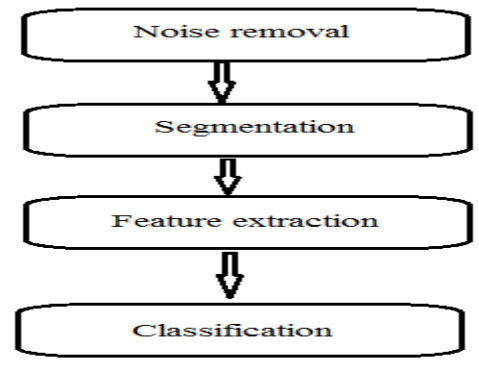

Fig.1 Architecture of proposed method

\section{RESULTS}

The images are collected from fetal ultrasound image gallery.

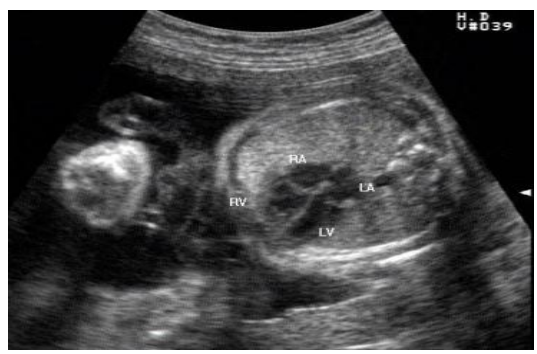

Fig 2. normal fetal heart

After collecting the image noise removal is done based on patch-based Wiener filter method. Figure show noisy and denoised image of normal fetal heart.

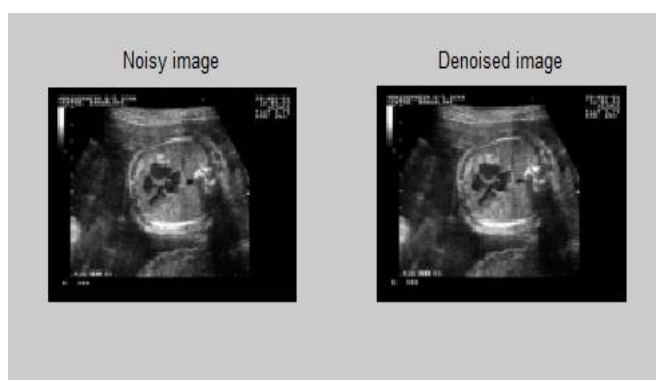

Fig 3. Noisy and denoised image

After that morphological opening operation is done using periodicline structuring element and figure show the result of morphological operation.

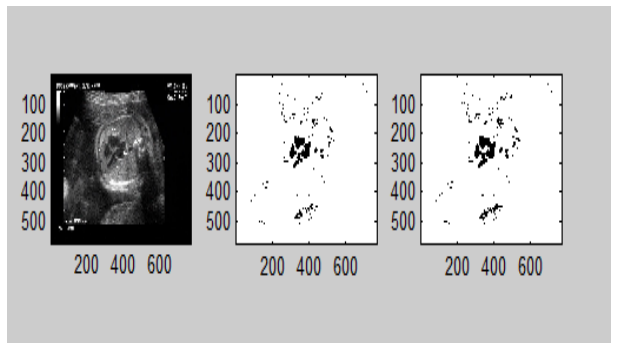

Fig 4. Morphological operation Using periodicline structuring element

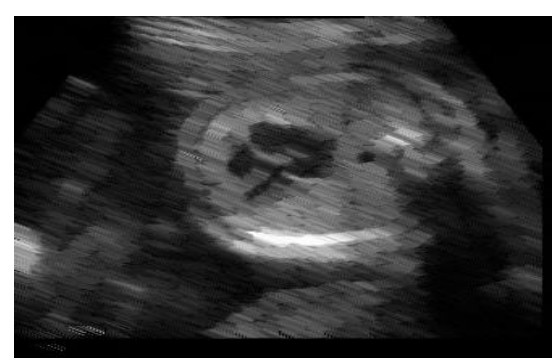

Fig 5. morphological opening

Next step is the feature extraction. Normalization, energy, correlation, sum of variances, inverse difference moment, entropy, correlation $1 \& 2$, sum average, sum entropy, sum variance, difference variance, contrast, difference entropy are the 13 features extracted from the image. 0.3248, 0.1745, $134.5739,4.7453,0.9360,3.6944,9.9363,1.5618,1.6765$, $0.0939,0.4065,-0.6704,0.9031$ are the values of 13 features Normalization, energy, correlation, sum of variances, inverse difference moment, entropy, correlation $1 \& 2$, sum average, sum entropy, sum variance, difference variance, contrast, difference entropy respectively of one image collected from ultrasound image gallery.

Final step is the classification based on this extracted features and display the result. During displaying result it display a confusion matrix and also plot performance

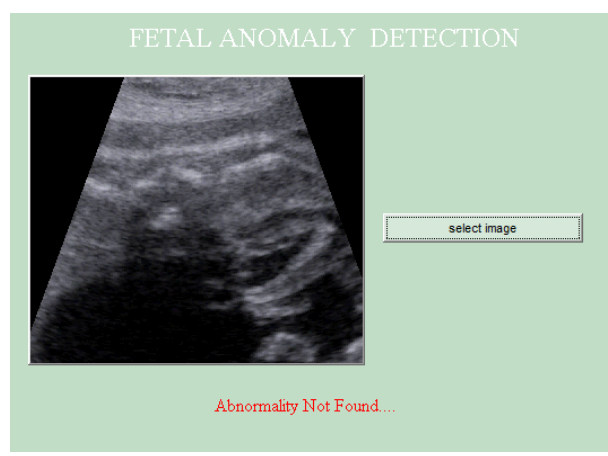

Fig 6. final result

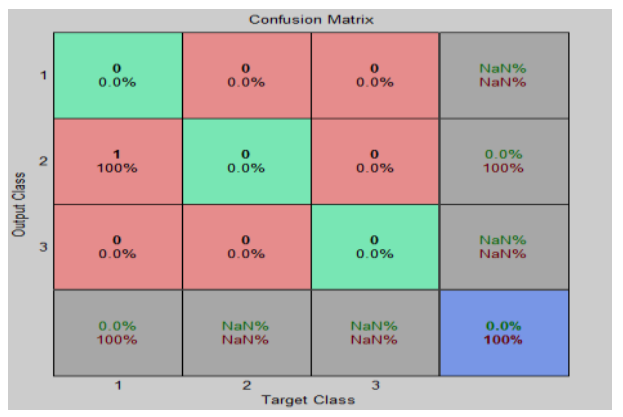

Fig 7 confusion matrix 
Table 2 . Overall classification details

\begin{tabular}{|c|l|}
\hline Number of images that are correctly classified & 30 \\
\hline Number of images that are not correctly classified & 9 \\
\hline
\end{tabular}

Table 3 . Classification details of each group

\begin{tabular}{|l|l|l|}
\hline $\begin{array}{l}\text { Classification } \\
\text { category }\end{array}$ & $\begin{array}{l}\text { Correctly } \\
\text { classified }\end{array}$ & $\begin{array}{l}\text { Incorrectly } \\
\text { classified }\end{array}$ \\
\hline Normal & 10 & 2 \\
\hline Defect in the valve & 15 & 5 \\
\hline Hole in the heart & 5 & 2 \\
\hline
\end{tabular}

\section{CONCLUSION}

Congenital heart defects are birth defects that affect the structure and function of the heart. These defects may be a hole in the heart or defect in the valve. If these defects are identify early then proper treat and care can give. Ultrasound image can be used to identify whether there is defect in the fetal during pregnancy period. Among all the defect in the fetal CHD is difficult to detect. Medical field relies on manual manipulation ultrasound images to make inferences about the presence of CHD. The chance of being seen the CHD from ultrasound image is nearly $25 \%$. The overall contribution of this project is the development of a system that can detect the presence of CHD using fetal echocardiographic images. One feature of ultrasound image is the presents of speckle noise. Removal of speckle noise is the first step. After that segmentation is done. Then 13 features are extracted from the segmented image using gray level co-occurrence matrix. Based on this feature classification is done. For that classification artificial neural network is used with 13 input neurons and 3 output neurons. 13 input neurons are corresponding to 13 features extracted from GLCM and 3 output neurons are corresponding to normal, hole in the heart and defect in the valve classes. Finally accuracy is calculated.

\section{REFERENCES}

[1] J A Noble "Ultrasound image segmentation and tissue characterization" Part H: J. Engineering in Medicine, Vol. 223, PP. 1-10, June 2009

[2] J. G. Bosch, S. C. Mitchell, B. P. F. Lelieveldt, F. Nijland, O. Kamp, M. Sonka, and J. H. C. Reiber, "Automatic segmentation of echocardiographic sequences by active appearance motion models," IEEE Trans.Med. Imag., vol. 21, no. 11, pp. 1374-1383, Nov. 2002

[3] N. Senthilkumaran and R. Rajesh, "Edge Detection Techniques for Image Segmentation - A Survey of Soft Computing Approaches", International Journal of Recent Trends in Engineering, Vol. 1, No. 2, May 2009.

[4] Y. Zimmer, S. Tepper, and R. Akselrod, "An automatic approach for morphological analysis and malignancy evaluation of ovarian masses using B-scans," Ultrasound Med. Biol., vol. 29, no. 11, pp. 1561-1570, 2003

[5] A. Bovik. On detecting edges in speckle imagery. IEEE Trans. Acoust., Speech, Signal Processing, 36(45):1618-1627, 1988
[6] G. DeVore, B. Siassi, and L. Platt. Fetal echocardiography. iv. m-mode assessment ofventricular size and contractility during the second and third trimesters of pregnancy in the normal fetus. Am J Obstet Gynecol, 150:981-988, 1984

[7] E. Dougherty. An Introduction to Morphological Image Processing. SPIE Optical Engineering Press, Bellingham, Wash., USA, 1992

[8] I. Dindoyal. Foetal Echocardiographic Segmentation. Phd dissertation, University College London, London, UK, 2009

[9] V. Ravi H.-J. Zimmermann "Fuzzy rule based classification with FeatureSelector and modified threshold accepting" Lehrstuhl Unternehmensforschung, RWTH, Templergraben 64, D-52056, Aachen, Germany Received 8 September 1998; accepted 22 December 1998

[10] I. Nedeljkovic "Image Classification Based On Fuzzy Logic" MapSoft Ltd, Zahumska 2611000 Belgrade, Serbia and Montenegro Commission VI, WG VI/1-3

[11] A. M. Khan, Ravi. S "Image Segmentation Methods: A Comparative Study “ ,International Journal of Soft Computing and Engineering (IJSCE) ISSN: 2231-2307, Volume-3, Issue-4, September 201384

[12] Ch. Hima Bindu and K. Satya Prasad “ An Efficient Medical Image Segmentation Using Conventional OTSU Method ",International Journal of Advanced Science and Technology Vol. 38, January, 2012

[13] Vanisree K, "Decision Support System for Congenital Heart Disease Diagnosis based on Signs and Symptoms using Neural Networks "International Journal of Computer Applications (0975 - 8887) Volume 19- No.6, April 2011

[14] R. Sivakumar "Comparative study of Speckle Noise Reduction of Ultrasound B-scan Images in Matrix Laboratory Environment" International Journal of Computer Applications (0975 - 8887) Volume 10- No.9, November 2010

[15] Marcelo de Carvalho Alves, Edson Ampélio Pozza João de Cássia do Bonfim Costa, Luiz Gonsaga de Carvalho , Luciana Sanches Alves ,"Adaptive neurofuzzy inference systems for epidemiological analysis of soybean rust", Environmental Modelling \& Software 26 1089-1096,2011.

[16] Aliaa A. A. Youssif, A. A. Darwish, and A. M. M. Madbouly, "Adaptive Algorithm for Image Denoising Based on Curvelet Threshold", IJCSNS International Journal of Computer Science and Network Security, VOL.10 No.1, January 2010.

[17] FU Z L. "Some New Methods for Image Threshold Selection." Computer Application, 2000,20(10):13-15.

[18] Ajala Funmilola A, Oke O.A, Adedeji T.O, Alade O.M, Adewusi E.A. "Fuzzy k-c-means Clustering Algorithm for Medical Image Segmentation" Journal of Information Engineering and Applications ISSN 22245782 Vol 2, No.6, 2012. 